\title{
PERSPECTIVAS DE MOBILIDADE URBANA SUSTENTÁVEL E A ADESÃO AO MODO CICLOVIÁRIO
}

\author{
Keully Crstynne Aquino Diógenes \\ Mestranda em Administração de Empresas \\ Instituto Federal do Ceará - IF Ceará \\ Universidade de Fortaleza - UNIFOR \\ keully.aquino@gmail.com \\ Maria Aurelina Farias de Araujo \\ Mestre em Administração de Empresas \\ Universidade de Fortaleza - UNIFOR \\ aurelinaf@hotmail.com \\ Mônica Mota Tassigny \\ Doutora em Educação \\ Universidade de Fortaleza - UNIFOR \\ monica.tass@gmail.com \\ Fabiana Pinto de Almeida Bizarria \\ Doutoranda em Administração \\ Universidade de Fortaleza - UNIFOR \\ bianapsq@hotmail.com
}

\section{RESUMO}

Diante dos conceitos de Mobilidade Urbana Sustentável, busca-se caracterizar a bicicleta como meio de transporte na direção de constituir perfil dos sujeitos usuários e levantar desafios à disseminação desse veículo como alternativa viável de locomoção. Para tanto, realizou-se pesquisa descritiva, com amparo na abordagem qualitativa por meio da análise de conteúdo, e quantitativa, com suporte em estatística descritiva e Análise de Cluster. Para análise, utilizou-se dados de 144 participantes, coletados por meio de questionário com perguntas fechadas e abertas. Identificou-se a formação de dois clusters, sendo que o cluster 2 indica maior percentual de jovens, de ambos os sexos, adeptos à bicicleta, o que evidencia maior conscientização desse público sobre questões socioambientais, em razão da difusão da temática nos últimos anos. Ainda quanto aos resultados, identificou-se os seguintes empecilhos à adesão: o comportamento dos motoristas (27\%); a falta de infraestrutura (25\%) e a violência urbana (17\%) foram os mais citados. Considera-se que a análise por cluster, numa abordagem compreensiva, contribui para a elaboração de políticas públicas para a Mobilidade Urbana Sustentável que considere a segmentação dos usuários, de forma que se projete ações mais aderentes à cada perfil, ou maior sensibilização com amparo nos valores presentes em cada cluster.

Palavras-chave: Bicicleta; Mobilidade urbana sustentável; Sustentabilidade.

\section{PROSPECTS OF SUSTAINABLE URBAN MOBILITY AND ADHESION TO CICLOVIÁRIO MODE}

\begin{abstract}
On the concepts of Sustainable Urban Mobility, the research seeks to characterize the bicycle as a means of transport, in the direction to form profile of the users and present challenges to the dissemination of this vehicle as a viable alternative mobility. Therefore, it was done descriptive research, with support in the qualitative approach through content analysis, and quantitative analysis, supported in descriptive statistics and Cluster Analysis. For analysis, we used data of 144 participants, collected through a questionnaire with closed and open questions. It was identified the formation of two clusters, and the cluster 2 indicates a higher percentage of young people of both sexes, fans of cycling, showing greater awareness of this public on environmental issues, because of the thematic spread in recent years. Still on the results, it identified the following obstacles to accession: the behavior of drivers (27\%); the lack of infrastructure (25\%) and urban violence (17\%) were the most cited. It is considered that the cluster analysis, a comprehensive approach, contributes to the development of public policies for Sustainable Urban Mobility which considers the segmentation of users, so that protrudes more adherent shares to each profile, or increased awareness in reliance upon the values present in each cluster.
\end{abstract}

Key words: Bicycle; Sustainable urban mobility; Sustainability. 
Perspectivas de mobilidade urbana sustentável e a adesão ao modo cicloviário.

\section{INTRODUÇÃO}

Questões relacionadas à mobilidade urbana têm se tornado foco de estudos e políticas em diferentes cidades no mundo, especificamente em decorrência do desenvolvimento econômico e social que impacta as necessidades de mobilidade nestes espaços e, também, pelo teor socioambiental que a mobilidade incita, haja vista a intensa poluição agregada aos veículos motorizados (Castro, Barbosa \& Oliveira, 2013).

Órgãos públicos, entidades sem fins lucrativos, iniciativas privadas e estudos acadêmicos se debruçam sobre a compreensão e análise da problemática, com vistas a levantar sugestões viáveis de mudanças significativas e que venham agregar qualidade de vida, de forma que a lógica do transporte motorizado individual seja discutida em função da coletividade nos espaços públicos (Castro et al., 2013; Gomide \& Morato, 2011).

Os investimentos públicos, com o intuito de disseminar a modalidade de transporte alternativo, têm sido intensificados nos últimos anos no contexto mundial (Ministério das Cidades, 2007). A cidade de Bogotá, por exemplo, reconhecida internacionalmente pelo avanço de transportes sustentáveis, investiu US $\$ 180$ milhões de dólares, no período de 1990 a 2002, em intervenções para enraizar a consciência do uso da bicicleta nos jovens e adultos. Ao mesmo tempo, os Estados Unidos da América investem o dobro desse valor anualmente em projetos que veiculam melhorias na mobilidade urbana (Hidalgo, 2011).

A mobilidade possibilitada pelo uso da bicicleta corresponde à tônica da Mobilidade Urbana Sustentável, à medida que colabora com a consciência sobre aspectos ambientais atrelada à economicidade da locomoção, com perfil não poluente, e, também, por cooperar com a difusão de práticas saudáveis por potencializar hábitos de locomoção que exigem o movimento dos sujeitos nas cidades (Castro et al., 2013).

Com o intuito de intensificar o uso da bicicleta em uma cidade, faz-se necessário estabelecer ações que desenvolvam a consciência do transporte alternativo em usuários de todas as classes sociais, bem como retomar a discussão sobre planejamento urbano, com a disponibilização de espaços para outros modelos de transporte (Pezzuto \& Sanches, 2004; Magalhães, Campos \& Bandeira, 2015). Ocorre, porém, a necessidade de aprofundamento acerca dos fatores que influenciam o uso da bicicleta e de como esses fatores sofrem variações, considerando os aspectos culturais dos sujeitos em contextos urbanos, a medida que pesquisas com essa finalidade podem contribuir com a adoção de políticas públicas nas cidades no escopo da Mobilidade Urbana Sustentável (Seabra, Taco \& Dominguez, 2013; Silveira \& Maia, 2015).

Diante disso, neste artigo busca-se caracterizar a bicicleta como meio de transporte, na perspectiva de constituir perfil dos sujeitos usuários e levantar desafios à disseminação desse veículo como alternativa viável de transporte. Para tanto, agruparam-se diferentes perfis de usuários de bicicleta como meio de transporte e categorizaram-se as principais dificuldades em empreender o uso da bicicleta no cenário da mobilidade urbana, na Cidade de Fortaleza, numa perspectiva descritiva, qualitativa e quantitativa de análise de dados coletados junto a 144 participantes.

A pesquisa, ainda, avança na discussão sobre o uso da bicicleta no cenário da mobilidade urbana nacional, enfatizando elementos de estudos recentes sobre a temática (Castro et al., 2013; Magalhães et al., 2015; Saldanha, Fraga \& Santos, 2015), bem como oferece a percepção sobre a bicicleta no cotidiano de um grande centro urbano e, por meio de clusters, organiza essa percepção em torno de perfis, o que contribui para melhor delimitar esses sujeitos-usuários e, com isso, especifica possibilidades de análise no campo das políticas públicas para a Mobilidade Urbana Sustentável que amplie a composição de propostas de engenharia, economia e planejamento urbano (Curtis \& Low, 2012), para agregar valores, cultura e sentimento de apropriação dos espaços urbanos (Bonfin, 2003; Costa, Ramos, Ramos, \& Oliveira, 2013), com referência nos impactos socioambientais atrelados à Mobilidade Urbana Sustentável (Azevedo Filho, 2012; Seabra et al., 2013). 


\section{A CULTURA E A MOBILIDADE URBANA}

O transporte nos grandes centros urbanos tem sido foco de estudos nacionais e internacionais (Amorim \& Serra, 2001; Castro et al., 2013; Bertini, 2005; Downs, 2004; Gomide, 2008; Magalhães et al., 2015; Plume, 2003; Ross \& Yinger, 2000; Saldanha et al., 2015; Zulkifli, Norma, Chong, \& Loh, 2001). Estes abordam desde os fatores considerados como responsáveis pelos congestionamentos e o desenvolvimento de infraestruturas que os reduza à necessidade de implantação de formas alternativas de transportes com o intuito de promover a mobilidade urbana sustentável e, também, o uso de bicicletas no âmbito do turismo.

Nas economias desenvolvidas ou em desenvolvimento ocorre o aumento da renda e do consumo dos indivíduos e, com isso, aumenta a possibilidade de aquisição do transporte individual motorizado, impactando a circulação nas áreas de intensa habitação (Dows, 2004; Gomide \& Morato, 2011; TCU, 2010). O individualismo, como tendência cultural, apresenta-se pela preferência ao transporte individual, reforçando o círculo vicioso do congestionamento e da expansão urbana (Castro et al., 2013; Roos \& Yinger, 2000). Atribui-se a essa cultura como um dos fatores responsáveis pelos problemas associados à mobilidade e, também, à existência de poucas iniciativas propositivas para a melhoria da circulação de pessoas nas cidades (Castro et al., 2013; Resende \& Sousa, 2009).

Por cultura entende-se o conjunto de pressupostos adquiridos coletivamente nas relações com meio ambiente e que serão repassados aos novos membros como a forma ideal a ser seguida (Heinzmann, Gubiani \& Scarpin, 2010). A cultura de um grupo representa um conjunto de comportamentos, normas e valores sociais aceitos e partilhados por todos os membros e que, de certa forma, os tornam únicos. Desta forma, ao considerar a cultura, percebe-se que não é possível uma explicação causal do fenômeno social e comportamental (Heinzmann, Gubiani \& Scarpin, 2010).

A bicicleta como meio de transporte, assim como seus benefícios para as cidades e população, possui maior expressão nos pequenos e médios centros urbanos e na periferia dos grandes centros urbanos, onde o menor fluxo de pessoas e transporte ainda impõe maior facilidade para a sua adesão (Ministério das Cidades, 2007; Vieira, Valente, Pegas, Moreira \& Oliveira, 2010). Por outro lado, cidades que apresentam a bicicleta como meio de transporte são mais eficientes do que as cidades que mantêm a cultura automobilística, não apresentam problemas de congestionamentos, os gastos do poder público com manutenção das vias são menores, apresentam redução na emissão de $\mathrm{CO} 2$ e menores gastos com saúde pública.

Ao mesmo tempo, o modo cicloviário possibilita que as cidades apresentem mobilidade urbana evoluída, permitindo aumento de espaços disponíveis nas vias e, com isso, melhoria do trânsito de pessoas e veículos. Pedalar possibilita ao cidadão ficar mais sensível ao que está a sua volta, uma vez que há menor poluição sonora causada pelos motores e maior contato com o ambiente, permitindo maior apropriação do espaço pelos ciclistas e pode, inclusive, gerar sentimentos de apego ao lugar, favoráveis a comportamentos socioambientais (Bomfim, 2003; Lynch, 1998; Moser, 2005), e por fim apresenta práticas mais intensivas de atividade física reduzindo os níveis de estresse (Barbieri, 1997).

Nesse contexto, a mobilidade urbana com base na bicicleta tem como objetivo disseminar a utilização desse meio de transporte para além das atividades de lazer. Contudo, compreender o comportamento dos indivíduos e avaliar os motivos que levam a utilizá-la diariamente não é tarefa simples, principalmente quando se observa que o automóvel assume a simbologia de status e prosperidade (Castro et al., 2013). Conhecer aspectos relacionados à adesão ao modo cicloviário é tema que pode contribuir para o aprofundamento dessas discussões, quando as questões culturais, ambientais e comportamentais ensejam proposições no tocante à sustentabilidade e à segurança, diante do cenário de mudanças climáticas, aumento do preço de combustíveis não renováveis e intenso congestionamento do tráfego nas cidades (Un-Habitat, 2013). 
Perspectivas de mobilidade urbana sustentável e a adesão ao modo cicloviário.

\subsection{Mobilidade urbana sustentável}

Para que se possa compreender a Mobilidade Urbana Sustentável, têm-se a sustentabilidade definida como a forma de conduzir a vida assegurando a sua continuidade no futuro, na perspectiva da coletividade e do bem-comum, sendo o desenvolvimento sustentável um processo de satisfação de necessidades com suporte na premissa de que essas necessidades devem ser supridas no futuro (Mebratu, 1998; Sinay, Dalbem, Loureiro, \& Vieira, 2013).

O termo desenvolvimento sustentável surgiu na década de 1980 quando foi citado no relatório "World Conservation Strategy: Living Resource for Sustainable Development", que teve como objetivo chamar atenção para a preservação da natureza (Silveira, 2010). Entretanto, somente a partir de 1987 é que esse termo obteve notoriedade mediante a Comissão Mundial sobre o Meio Ambiente e Desenvolvimento, conhecida como Comissão Brundtlan, que produziu o Relatório Brundtlan com o título "O Nosso Futuro Comum" e onde se encontra a definição que contém os princípios que fundamentam e que compuseram a base da Agenda 21, aprovada por mais de 180 países por ocasião da realização da Conferência da ONU sobre o Meio Ambiente e Desenvolvimento, realizada no Rio de Janeiro em 1992 (Hopkins, 2012; Sulaiman, 2011).

Desde os anos 1990, acadêmicos e profissionais têm argumentado que para as empresas parecerem sustentáveis, devem, simultaneamente, atender às dimensões: ambiental, social e econômica (Costa et al., 2013; Montiel, 2008).

No Brasil, o Ministério dos Transportes adota como referência os preceitos do desenvolvimento sustentável para a evolução de sua política ambiental, contribuindo para que as gerações futuras possam usufruir da base de recursos naturais disponíveis no país (Ministério dos Transportes, 2007). Nesse sentido, há necessidade de planejamento e, principalmente, da anuência e do reconhecimento de que os recursos naturais são finitos (Banerjee, 2008). Depende, ainda, da adoção de atitudes que contribua para um desenvolvimento mais sustentável para as cidades e essa perpassa por mudanças culturais e de valores (Costa et al., 2013). O uso racional de automóveis, a adoção de políticas públicas, o investimento em transporte coletivo e o fomento ao uso de transporte alternativo, como a bicicleta, são algumas destas ações (Silveira, 2010).

As cidades estão revendo seus modelos e descobrindo alternativas e estratégias sustentáveis de crescimento (Magalhães et al., 2015). Uma metrópole depende de dois fatores básicos para funcionar: um bom planejamento de uso do solo, somado a um sistema de transporte eficiente (Mitcheel, 2008). Dessa forma, compreende-se que o padrão de consumo nas cidades é que vai determinar o que irá acontecer ao planeta (Hillel, 2008).

A mobilidade urbana é composta pelas interações oriundas dos deslocamentos de pessoas e bens, ou seja, o conceito de mobilidade urbana vai além dos simples deslocamentos de veículos, motorizados ou não, que são utilizados para este fim (Ministério das Cidades, 2007). Ela compreende fatores que influenciam a qualidade de vida das pessoas por melhorar o acesso a serviços essenciais, contribuindo com a qualidade de vida e formação de estilos de vida capazes de alterar padrões de adoecimento. A urbanização, ainda, deve ser considerada um processo social no qual diferentes atores podem interagir com diferentes agendas com práticas espaciais particulares, porém interligadas (Oliveira \& Misoczky, 2016).

Ao mesmo tempo, o trânsito nas cidades tem sido marcado pelo uso de muitos meios de transporte poluentes, com emissão de gases de efeito estufa, intensos congestionamentos, ruídos, e emergência de riscos à população por impactar na sua saúde e segurança. Portanto, compreende-se a Mobilidade Urbana Sustentável, como resultado do deslocamento de pessoas nos centros urbanos com menor impacto ao meio ambiente e, consequentemente, maior probabilidade de ampliação da qualidade de vida e manutenção da vida no futuro (Seabra et al., 2013).

Bicicletas favorecem o desenvolvimento da Mobilidade Urbana Sustentável ao representarem uma forma de transporte que apresenta benefícios para as cidades e para o cidadão. Este, ao pedalar, é estimulado a desenvolver hábitos diferenciados, influenciados pela rotina cada vez mais intensa de trabalho, mediada pela constante necessidade de incorporar estratégia para 
agregar saúde à vida. Por essas razões, cidades oriundas tanto de países desenvolvidos quanto de países emergentes incentivam o modo cicloviário como forma de melhorar a mobilidade urbana (Vieira et al., 2010).

Entretanto, apesar de ser um meio de transporte não poluente, de baixo custo de aquisição e manutenção e que traz benefícios para a saúde do usuário, somente $7 \%$ dos brasileiros utilizam a bicicleta como principal meio de transporte, de acordo com dados do Sistema de Indicadores de Percepção Social sobre mobilidade urbana, divulgados pelo Instituto de Pesquisa Econômica Aplicada (Ipea, 2011). Pode-se constatar que "[...]isso se deve em parte à pequena importância dada ao potencial dos veículos de tração humana, já que a maioria das cidades não possui infraestrutura adequada para a circulação segura e confortável do seu usuário" (Castro et al., 2013, p. 149).

No sentido de melhorar a mobilidade urbana, o Ministério das Cidades implementou, via Secretaria de Transporte e da Mobilidade Urbana [SeMob], o Programa Brasileiro de Mobilidade por Bicicleta, conhecido por Bicicleta Brasil. De abrangência nacional, o programa incentiva o uso da bicicleta integrada com os transportes coletivos. Uma de suas principais ações é estimular inclusive via financiamento - os governos municipais a implementarem sistemas cicloviários e ações que garantam a segurança de ciclistas (Ministério das Cidades, 2007).

Com a promulgação da Lei $n^{\circ} 12.587 / 12$, que instituiu as diretrizes da Política Nacional de Mobilidade Urbana, o Programa Bicicleta Brasil teve um novo avanço, pois a lei tornou obrigatória a elaboração, até 2015, de Planos de Mobilidade Urbana [PMU] para todos os municípios com mais de 20 mil habitantes. Os principais pontos destacados na Política Nacional de Mobilidade Urbana estão assim descritos:

1. Prioridade do transporte não motorizado e dos serviços públicos coletivos sobre o transporte individual motorizado;

2. Restrição e controle de acesso e de circulação, permanentes ou temporários, de veículos motorizados em locais e horários predeterminados;

3. Estabelecimento de padrões de emissão de poluentes para locais e horários determinados;

4. Possibilidade de cobrar pela utilização da infraestrutura urbana, para desestimular o uso de determinados modos e serviços de mobilidade;

5. Dedicação de espaço exclusivo nas vias públicas ao transporte público coletivo e a modos de transporte não motorizados, como as bicicletas;

6. Inclusão do usuário no planejamento, na fiscalização e na avaliação da política local de mobilidade urbana (Soares, Guth, Amaral, \& Maciel, 2015).

Desta forma, a redução da utilização de veículos motorizados, especialmente automóveis e motos, tornou-se condição necessária para alcançar a Mobilidade Urbana Sustentável (Silveira, 2010), como forma de mitigar impactos socioambientais negativos advindos da cultura do transporte motorizado individual, bem como ampliar a discussão dos espaços públicos que viabilizem conduções alternativas, como a bicicleta.

\subsection{Fatores relacionados à adesão ao modo cicloviário}

A disseminação do uso da bicicleta como meio de transporte é considerada como o marco inicial para a melhoria da mobilidade nas grandes cidades (Castro et al., 2013). Esse modelo exige menor investimento privado e público, com expectativa de utilização de pouco espaços públicos, e permite maior agilidade, quando comparado com outros modos de transporte.

Nos estudos de Pezutto e Sanches (2004), realizados em uma cidade brasileira de médio porte, foram identificados fatores que influenciam a escolha da bicicleta como meio de transporte, com amparo da distinção entre ciclistas, ciclistas casuais e não ciclistas. Esse estudo concluiu que o fator conforto apresenta a maior relevância para os três grupos, seguido do fator segurança.

A pesquisa realizada por Providelo e Sanches (2010), identificou percepções de indivíduos acerca do uso da bicicleta em diversas instituições ligadas à educação, em duas cidades do interior 
de São Paulo, sendo que o fato de a bicicleta ser um transporte barato e bom para a saúde foram as considerações mais ressaltadas pelos entrevistados.

Maia e Moreira (2010), em pesquisa realizada em Fortaleza visando retratar a realidade dos usuários de bicicleta na cidade, concluíram que os ciclistas entrevistados identificam a insegurança quanto à ocorrência de acidentes e assaltos. Entretanto, um dos principais fatores de dificuldades relatados foi a falta de educação no trânsito por condutores de transporte motorizado, e, como sugestão de melhoria, foi citada a construção de mais ciclovias na cidade.

Os estudos de Silveira e Maia (2015) apresentam os fatores que explicam o perfil dos usuários das bicicletas no exterior e no Brasil, com o objetivo de compreender os fatores motivadores e os empecilhos no uso desta modalidade de transporte. Os fatores foram levantados em artigos nacionais de anais de congresso e periódicos e em teses e dissertações nacionais no intervalo de 2000 a 2013. A pesquisa teve como objetivo apresentar um panorama das variáveis que influenciam o uso da bicicleta no Brasil com amparo na Teoria do Comportamento Planejado. Os fatores motivadores mais mencionados nas pesquisas realizadas nas cidades brasileiras foram: andar de bicicleta é bom para a saúde (citados em 5 cidades); baixo custo da bicicleta (3 cidades) e redução de tempo de deslocamento (3 cidades). Os empecilhos mais citados foram: insegurança no trânsito (citados em 14 cidades); falta de vias cicláveis ( 9 cidades) e imperfeição na pavimentação, citadas em 7 cidades (Silveira \& Maia, 2015).

Analisando o modelo proposto na pesquisa desenvolvida por Silveira e Maia (2015), criado para explicar o uso da bicicleta no exterior, foram identificados 21 fatores motivadores e 15 empecilhos. As pesquisas foram realizadas em países distintos para compreender qual o papel de cada variável e determinar a sua importância. Como resultado, a pesquisa revelou a variável acessibilidade e infraestrutura como a mais citada entre os fatores motivadores e a falta de infraestrutura cicloviária continuada foi a mais citada como empecilho. Os aspectos econômicos para aquisição de veículos automotores foram considerados como menos relevantes.

\section{METODOLOGIA}

Segundo Franco (2012), a região nordeste concentra 26\% dos usuários de bicicleta no Brasil. A cidade de Fortaleza, capital do Estado do Ceará, apresenta uma evolução populacional crescente, com uma população estimada, em 2014, em 2.571.896 habitantes (PMF, 2015). Apresenta uma área territorial de $314,930 \mathrm{~km}^{2}$, tendo $7.786,44 \mathrm{hab} / \mathrm{km}$. Possui características geográficas com relevo litorâneo com poucas rampas e altitude média de 15 metros. O clima tem média anual de $26^{\circ}$ e as chuvas são concentradas entre os meses de março e maio, com média pluviométrica de $1.467,1$ $\mathrm{mm} /$ ano. A frota de veículos motorizados é de 964.724 veículos, sendo composta por 536.895 carros (Soares et al., 2015).

A figura 1 mostra que grande parcela da população de Fortaleza reside em locais afastados do centro comercial. 


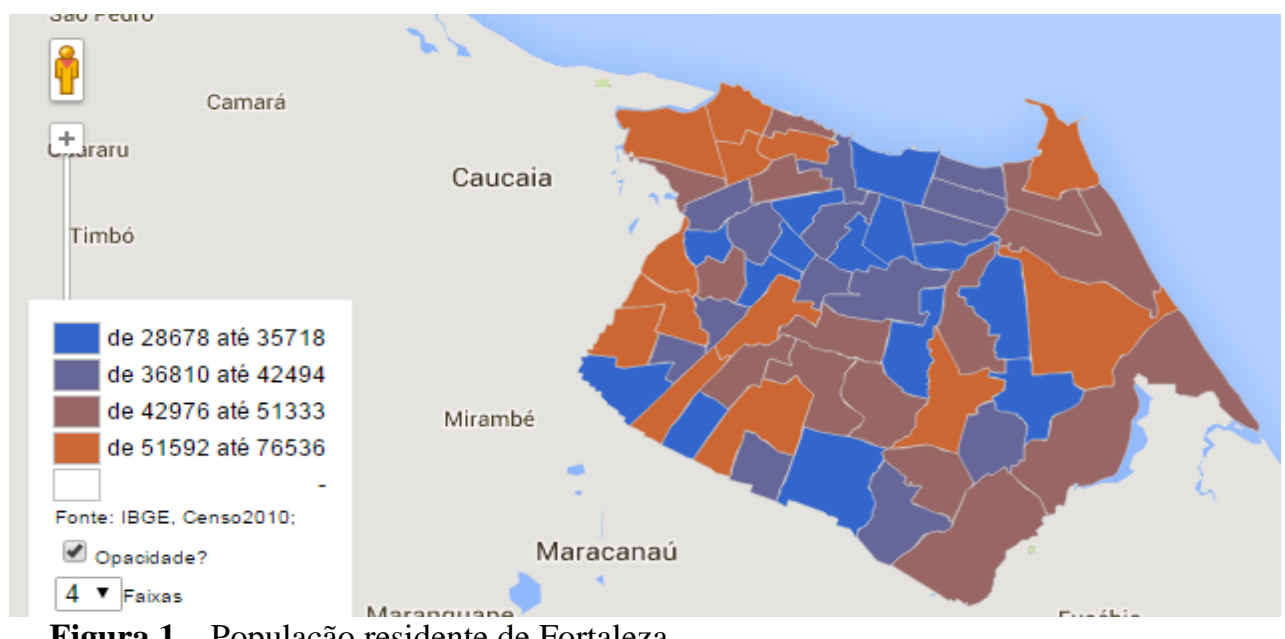

Figura 1 - População residente de Fortaleza

Fonte: Ibge (2010)

A figura 2 expõe a concentração comercial da cidade, demonstrando seu caráter centralizado, o que faz com que a cidade sofra com uma rotina de congestionamentos, principalmente nos horários que coincidem como início e o término do expediente comercial.
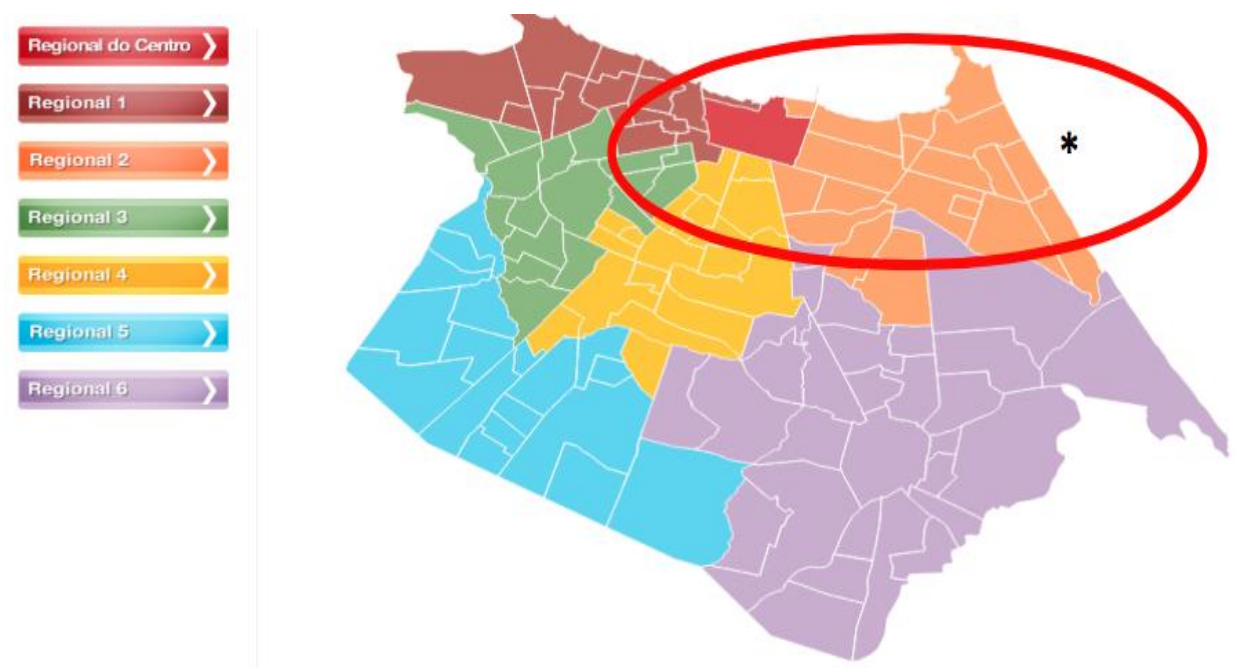

Figura 2 - Região que apresenta a maior concentração comercial

Fonte: Adaptada de Prefeitura Municipal de Fortaleza (2015)

Nona: * O círculo indica a região de maior concentração comercial

A pesquisa possui a combinação da abordagem quantitativa e qualitativa numa perspectiva de triangulação, o que a torna mais forte e reduz os problemas gerados pela adoção de um único método (Maxwell, 1996; Azevedo, Oliveira, Gonzalez \& Abdalla, 2013). A triangulação teve o objetivo de empreender a formação de clusters de usuários de bicicleta como meio de transporte, bem como levantar aspectos relacionados às dificuldades de disseminação desse veículo como alternativa viável de transporte. Para tanto, realizou-se pesquisa de campo exploratória entre os meses de maio e junho de 2015, com coleta de dados por meio de um questionário contendo informações sociodemográficas (informações econômicas, idade, gênero e o uso da bicicleta como meio de transporte) e uma questão aberta, destinada a captar três fatores considerados como empecilhos ao uso da bicicleta como meio de transporte.

O questionário eletrônico foi aplicado utilizando-se a ferramenta Google Drive, disponibilizado pela internet com divulgação em redes sociais dos pesquisadores. Participaram da 
pesquisa 144 pessoas, amostra aleatória por acessibilidade, de adultos residentes na Cidade de Fortaleza que afirmaram fazer uso de bicicletas no seu cotidiano, seja como veículo de locomoção diário, ou de forma esporádica em atividades de lazer.

Com amparo na pesquisa quantitativa, realizou-se estatística descritiva e Análise de Cluster [AC]. No caso deste, realizou-se análise AC, com amparo no método $K$-Means utilizado para gerar o agrupamento de casos com a finalidade de apreender grupos que possuem características comuns, com grande similaridade interna e dissimilaridade externa, com base no centroide do grupo de variáveis escolhidas. O método é adequado quando se conhece, a priori, a quantidade de agrupamentos para análise do estudo (Pohlmann, 2009). Nesse caso, a solução bipartite foi escolhida, por contemplar diferenças entre dois perfis de adeptos ao modo de transporte cicloviário. Por ser um método não hierárquico, a técnica "[...]atribui objetos a um grupo uma vez que o número de grupos a ser formado esteja especificado. Dessa forma, a solução de seis grupos não é uma combinação de soluções de dois a sete grupos, mas é baseada apenas no encontro da melhor solução de seis grupos" (Pohlmann, 2009, p. 348)

No que diz respeito à análise qualitativa, empreendeu-se estudo das questões consideradas como empecilhos ao uso da bicicleta como meio de transporte, com suporte na Análise de Conteúdo, permitindo a inferência de conhecimentos relativos às condições de produto/recepção (variáveis inferidas) dessas mensagens (Bardin, 2011).

Para organização, categorização e apreciação dos dados utilizou-se o software Spss, para análise descritiva e de agrupamento (Análise de Cluster) e, também, Atlas-TI, especificamente para auxiliar a categorização dos empecilhos ao uso de bicicleta e ilustrar as palavras mais citadas pelos sujeitos do estudo, como informação complementar à categorização.

\section{APRESENTAÇÃO E ANÁLISE DOS DADOS}

\subsection{Características dos participantes}

Em termos de gênero, verificou-se que homens $(58,33 \%)$ e mulheres $(41,66 \%)$ estão quase que igualmente representados na amostra (figura 3). Os respondentes com idades de 18 a 30 anos, respondem por $45,83 \%$ da amostra, e $36,11 \%$ dos participantes possuem pós-graduação, sendo $24 \%$ especialistas, $16 \%$ mestres e $2 \%$ doutores.

\begin{tabular}{|l|l|l|}
\hline Sujeitos da pesquisa & Quantidade & Percentual \\
\hline Homens & 84 & $58,33 \%$ \\
\hline Mulheres & 60 & $41,66 \%$ \\
\hline Entre 18 e 30 anos & 66 & $45,83 \%$ \\
\hline Entre 31-61 anos & 78 & $54,16 \%$ \\
\hline Segundo grau completo & 4 & $2,7 \%$ \\
\hline Superior incompleto & 49 & $34,02 \%$ \\
\hline Superior completo & 39 & $27,08 \%$ \\
\hline Pós-graduação & 52 & $36,11 \%$ \\
\hline Uso de bicicleta & 93 & $64,58 \%$ \\
\hline Não uso de bicicleta & 51 & $34,41 \%$ \\
\hline
\end{tabular}

Figura 3: Características dos participantes

Fonte: Dados da pesquisa (2015) 
Cerca de $39 \%$ dos participantes declararam ter cursado o ensino superior, enquanto apenas 2,7\% declaram ter cursado somente até o ensino médio. Pelos dados apurados, pode-se inferir que a maior parte dos participantes é jovem e possui nível educacional elevado.

Sobre o uso de bicicleta, 64,58\% dos respondentes que afirmaram serem usuários da bicicleta como meio de transporte em contrapartida aos $34,41 \%$ que não são usuários desta modalidade.

\subsection{A formação dos clusters}

No que tange à composição dos clusters, utilizou-se o método K-Means, inicialmente com base na variável uso de bicicleta. Tendo como definição para o estudo, a formação de dois grupos com as variáveis idade, gênero, perfil ocupacional, escolaridade, observa-se que há maior concentração de casos no cluster 2, com 96 casos, em comparação com o cluster 1, com 48 participantes, o que deve ser considerado na análise, haja vista a leitura proporcional das variáveis em cada cluster.

Na tabela 1 identifica-se a composição da idade nos clusters. No grupo 1, composto por 48 participantes, há idades menos concentradas ao longo do continuum 18-61 anos, inclusive com a participação das idades 58 e 61 anos. Por sua vez, o grupo 2 tem a participação de 96 casos, cujas idades possuem maior distribuição entre 20 a 35 anos. Dessa forma, no que diz respeito à idade infere-se que há maior participação do público jovem no cluster 2.

Tabela 1: Distribuição da idade por Cluster

\begin{tabular}{|c|c|c|c|c|c|c|c|c|c|c|c|c|c|c|c|c|c|c|c|c|c|c|c|c|c|c|c|c|}
\hline & \multicolumn{26}{|c|}{ Idade } & \multirow[t]{2}{*}{ Total } \\
\hline & & 18 & 19 & 20 & 24 & 25 & 26 & \begin{tabular}{l|l}
27 & 2
\end{tabular} & \begin{tabular}{l|l}
28 & 29
\end{tabular} & 30 & 31 & $32 ?$ & \begin{tabular}{l|l}
33 & 3
\end{tabular} & \begin{tabular}{l|l}
34 & 35
\end{tabular} & \begin{tabular}{l|l}
35 & 36
\end{tabular} & \begin{tabular}{|l|}
37 \\
\end{tabular} & 38 & \begin{tabular}{|l|l}
39 & 4
\end{tabular} & \begin{tabular}{l|l}
40 & 4
\end{tabular} & \begin{tabular}{l|l}
41 & 4.
\end{tabular} & \begin{tabular}{l|l}
43 & 44
\end{tabular} & \begin{tabular}{l|l}
4 & 45
\end{tabular} & \begin{tabular}{|l|l|}
5 & 47 \\
\end{tabular} & \begin{tabular}{|l|}
53 \\
\end{tabular} & 57 & \begin{tabular}{|l|}
58 \\
\end{tabular} & 61 & \\
\hline Cluster & 1 & 0 & 0 & 0 & 3 & 4 & \begin{tabular}{l|l}
2 & 2 \\
\end{tabular} & \begin{tabular}{l|l}
2 & 6 \\
\end{tabular} & \begin{tabular}{l|l|}
6 & 2 \\
\end{tabular} & 4 & 2 & \begin{tabular}{l|l}
3 \\
\end{tabular} & \begin{tabular}{l|l}
1 & 1 \\
\end{tabular} & \begin{tabular}{l|l}
1 & 4 \\
\end{tabular} & 42 & 1 & 1 & 0 & 0 & \begin{tabular}{l|l|}
1 & 3 \\
\end{tabular} & \begin{tabular}{|l|l|}
3 & 2 \\
\end{tabular} & 1 & 0 & 0 & 0 & 1 & 1 & 48 \\
\hline Cluster & 2 & 1 & 2 & 2 & 7 & 6 & 6 & \begin{tabular}{l|l}
4 & 1
\end{tabular} & \begin{tabular}{l|l}
11 & 2
\end{tabular} & 4 & 3 & \begin{tabular}{l|l}
5 & $?$
\end{tabular} & \begin{tabular}{l|l}
3 & 1
\end{tabular} & \begin{tabular}{l|l}
1 & 5
\end{tabular} & \begin{tabular}{l|l}
5 & 1 \\
\end{tabular} & 1 & \begin{tabular}{l|l}
0 \\
\end{tabular} & \begin{tabular}{l|l}
1 & 1 \\
\end{tabular} & \begin{tabular}{l|l}
1 & 2 \\
\end{tabular} & \begin{tabular}{l|l}
2 & 2 \\
\end{tabular} & \begin{tabular}{l|l}
2 & 0 \\
\end{tabular} & 0 & 1 & 2 & 1 & 0 & 0 & 96 \\
\hline Total & & & & & & & & & & & & & & & & & & & & & & & & & & & & 144 \\
\hline
\end{tabular}

Fonte: Dados da pesquisa (2015), com auxílio do software SPSS 22

A formação dos grupos tendo como variável "gênero" teve a distribuição de acordo com a tabela 2. Percebe-se que há maior percentual de homens no cluster 1, haja vista que há dois homens para cada mulher (32/16). Da mesma forma, observa-se que o cluster 2 possui um percentual masculino menor em comparação ao cluster 1 , considerando que há 1,18 homens para cada mulher $(52 / 44)$.

Tabela 2: Distribuição do gênero por Cluster

\begin{tabular}{|c|c|c|c|c|}
\hline & & \multicolumn{2}{|c|}{ Cluster } & \multirow[t]{2}{*}{ Total } \\
\hline & & 1 & 2 & \\
\hline \multirow{2}{*}{ Gênero } & $1(\mathrm{~F})$ & \multicolumn{2}{|c|}{16} & 60 \\
\hline & $2(\mathrm{M})$ & \multicolumn{2}{|c|}{32} & 84 \\
\hline \multicolumn{2}{|l|}{ Total } & \multicolumn{2}{|c|}{48} & 144 \\
\hline
\end{tabular}

O comportamento dos perfis ocupacionais nos dois clusters é identificado na tabela 3 . Há maior concentração de funcionários públicos e professores no cluster 1, em comparação com cluster 2 que, além de possuir maior participação de estudantes, possui perfil mais diversificado de profissões. 
Tabela 3: Distribuição do perfil ocupacional por Cluster

\begin{tabular}{lc|l|r}
\hline \multicolumn{1}{c}{ Ocupação } & $*$ & \multicolumn{1}{c}{ Ocupação } & $*$ \\
\hline Físico & 1 & Administrador & 4 \\
Fotógrafa & 1 & Advogada & 6 \\
Funcionário Público & 10 & Analista de Negócios & 2 \\
Gerente & 1 & Analista de Sistemas & 6 \\
Gestor cultural & 1 & Analista Judiciário & 1 \\
Ilustrador & 1 & Arquiteto e Urbanista & 3 \\
Mecânico & 1 & Artista & 4 \\
Musico & 1 & Assistente administrativo & 9 \\
Oceanógrafo & 1 & Assistente Social & 3 \\
Procurador & 1 & Autônomo & 1 \\
Professor & 16 & Bancário & 1 \\
Programador & 2 & Blogueira & 1 \\
Psicóloga & 1 & Estudante & 37 \\
Publicitária & 4 & Empresário & 4 \\
Recepcionista & 1 & Contador & 1 \\
Revisor & 1 & Coordenadora de eventos & 1 \\
Secretária & 2 & Designer & 4 \\
Técnico de informática & 1 & Economista & 1 \\
& & Engenheiro & 5 \\
& & Estagiária & 3 \\
\hline
\end{tabular}

Fonte: Dados da pesquisa (2015), com auxílio do software SPSS 22.

* Quantidade de pessoas por perfil ocupacional

Em relação à escolaridade, os grupos, representados na tabela 3, possuem a seguinte configuração. O cluster 1 possui distribuição de escolaridade menos concentrada, tendo respondentes de todos os níveis de escolaridade. No cluster 2, por sua vez, há predomínio de respondentes que possuem curso superior incompleto, completo e, também, curso em nível de especialização.

Tabela 4: Distribuição da escolaridade por Cluster

\begin{tabular}{ll|r|r|r}
\hline & & \multicolumn{2}{c|}{ Cluster } & \multicolumn{2}{c}{ Total } \\
\cline { 3 - 4 } & Superior incompleto & \multicolumn{2}{c}{$\mathbf{2}$} & \\
\hline & Superior completo & 10 & $\mathbf{4 1}$ & 49 \\
Grau de escolaridade & Segundo grau completo & 2 & 2 & 39 \\
& Especialista & 13 & $\mathbf{1 5}$ & 4 \\
& Mestre & 11 & 9 & 28 \\
Total & Doutor & 4 & 0 & 20 \\
& & $\mathbf{4 8}$ & $\mathbf{9 6}$ & $\mathbf{1 4 4}$ \\
\hline
\end{tabular}

Fonte: Dados da pesquisa (2015), com auxílio do software SPSS 22

Por fim, para a caracterização de cada cluster identifica-se a utilização de bicicleta como meio de transporte (Tabela 5). Nesse caso, o cluster 1 possui percentual maior de afirmações sobre a questão, haja vista que 1,66 pessoas para cada 1 (30/18) respondente afirmam fazer uso da bicicleta. No cluster 2 também há maior participação de respondentes usuários, porém, numa proporção maior, no caso, na proporção de 1,9 para cada 1 não usuário. 
Tabela 5: Uso de bicicleta por cluster

\begin{tabular}{|c|c|c|c|c|}
\hline & & \multicolumn{2}{|c|}{ Cluster } & \multirow[t]{2}{*}{ Total } \\
\hline & & 1 & 2 & \\
\hline Você utiliza bicicleta como meio de & $1(\mathrm{Sim})$ & \multicolumn{2}{|c|}{30} & 93 \\
\hline transporte? & 2 (Não) & \multicolumn{2}{|c|}{18} & 51 \\
\hline Total & & \multicolumn{2}{|c|}{48} & 144 \\
\hline
\end{tabular}

Fonte: Dados da pesquisa (2016), com auxílio do software SPSS 22

Com base na formação dos clusters e na distribuição das variáveis nesses grupos (figura 2, tabelas 2, 3, 4 e 5), infere-se o perfil dos dois clusters. O primeiro, com predomínio de respondentes do sexo masculino, é formado por pessoas menos jovens em comparação com o cluster 2. Além disso, possui sujeitos que trabalham em empregos mais estáveis (servidores públicos) e tradicionais (professores) com representantes de todos os níveis de formação. Nesse grupo, há menor participação de usuários de bicicleta como meio de transporte.

O cluster 2 é formado por uma maior participação feminina em comparação com o cluster 1 , possui um maior número de jovens com perfil profissional mais diversificado e com predomínio da formação em nível superior (completo e incompleto) e especialização. Nesse caso, há maior utilização de bicicletas como meio de transporte.

Infere-se, com amparo nos dados da pesquisa, que o perfil dos clusters indica uma possível evolução na discussão sobre uso de bicicletas, impactando o público mais jovem, com a migração de um perfil masculino para um perfil mais equilibrado entre homens e mulheres. O público mais jovem, também, expressa a formação em nível superior incompleto com a presença de muitos estudantes e, também, completo, com a participação de variadas profissões.

Considera-se que o perfil do cluster 2 pode ser reflexo de políticas emergentes para a melhoria da mobilidade nas grandes cidades (Castro et al., 2013), agregando maior conscientização por parte de um público ainda em formação e em processo de construção identitária, no que diz respeito à profissionalização e à participação social. A esse grupo também se atribui maior difusão de conhecimento, com uso intenso das novas tecnologias para acesso a informação, o que amplia a possibilidade de conhecer as problemáticas urbanas e, também, aderir a novos conceitos de vida, por esta ainda estar sendo elaborada em função dos valores em construção (Costa et al., 2013; Montiel, 2008).

A ampliação da premissa da sustentabilidade pode, também, ter contribuído com esse resultado, pois a discussão das questões ambientais são marca dos anos 1990 (Sulaiman, 2011; Hopkins, 2012), com evolução no campo educacional por meio da difusão de novos valores socioambientais (Albuquerque, Vicentini \& Pipitone, 2015; Valdanha \& Kawasaki, 2015), que marcam uma nova ética ambiental (Viveiro et al., 2015).

\subsection{Empecilhos ao uso da bicicleta como meio de transporte}

Segundo a Prefeitura Municipal de Fortaleza, em 2015, existem $120 \mathrm{~km}$ de ciclovias na cidade e a meta é ampliar o Programa de Expansão da Malha Cicloviária que prevê a implantação de novos $100 \mathrm{~km}$ de ciclovias, ciclofaixas e ciclorotas até agosto de 2016, de acordo com o Plano Diretor Cicloviário Integrado [Pdci], aprovado em dezembro de 2014 (PMF, 2015). A título de comparação, o Sistema Cicloviário da cidade de São Paulo apresenta 219,5 km de ciclovias, 3,3 km de ciclofaixas permanentes e 67,5 km de ciclorotas em 2015 (Soares et al., 2015), o que demonstra que Fortaleza pretende avançar no que diz respeito ao uso de bicicletas, embora ainda não apresente estrutura comparável a São Paulo.

Ao mesmo tempo, ressalta-se que somente investir na construção de ciclofaixas, ciclovias e ciclorotas não garante a disseminação do uso da bicicleta (Ary, 2009). O indivíduo precisa identificar a bicicleta como um veículo e não somente como um objeto de lazer, caso contrário as ciclofaixas transformam-se em problemas (Bezerra, Kaiser \& Battistelle, 2015). 
Na figura 4, são apresentadas 12 categorias relacionadas às percepções dos participantes em resposta à identificação de fatores considerados como empecilhos ao uso da bicicleta como meio de transporte. Os principais desafios são: o comportamento dos motoristas (27\%); falta de infraestrutura (25\%); violência urbana (17\%). Estes 3 aspectos correspondem a $69 \%$ das percepções mais citadas pelos fortalezenses.

Resultado semelhante foi identificado no estudo de Maia e Moreira (2010), que indica o comportamento de motoristas na cidade de Fortaleza como aspecto que incita resistência à adoção da bicicleta como meio de transporte.

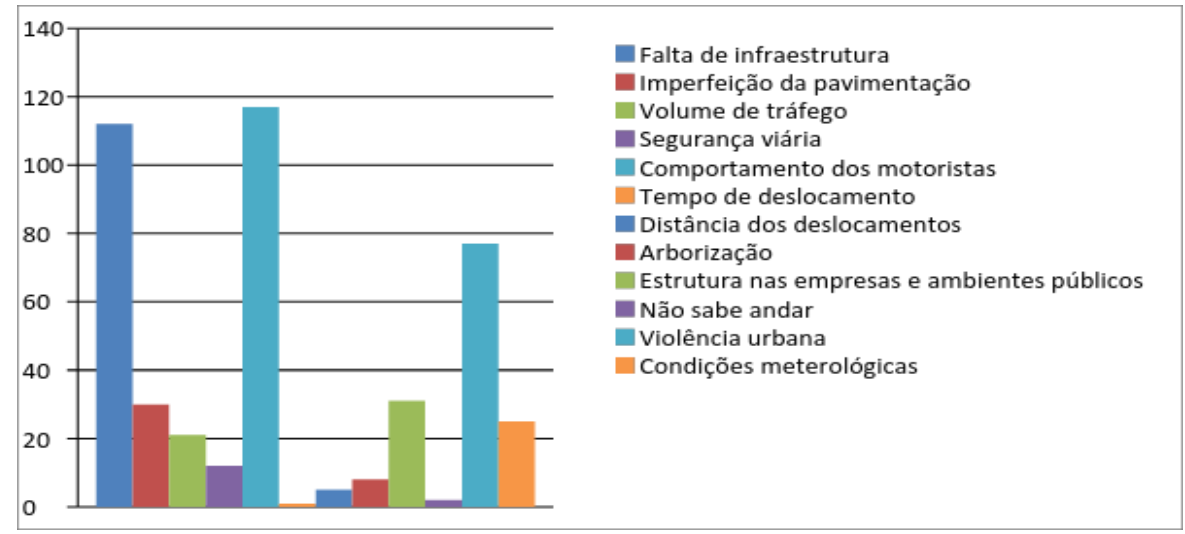

Figura 4: Variáveis identificadas como empecilhos

Fonte: Dados da pesquisa (2015)

A figura 4 também apresenta elementos que podem ser analisados em comparação aos principais pontos destacados na Política Nacional de Mobilidade Urbana citados por Soares et al. (2015), pertinente à variável falta de infraestrutura que teve $25 \%$ de citações, pode-se inferir que ainda falta dar prioridade ao transporte não motorizado. Quanto à violência urbana que obteve $17 \%$ de citações, os dados corroboram com os estudos de Maia e Moreira (2010) que concluíram que a insegurança quanto à ocorrência de acidentes e assaltos foi um dos itens mais relacionados na pesquisa. A variável estrutura nas empresas e ambientes públicos alerta para o que Montiel (2008) ressalta quando trata das empresas consideradas sustentáveis que, para assim parecerem, devem atender às dimensões ambiental, social e econômica.

Para complementar a abordagem das dificuldades mencionada, a tabela 6 ilustra a frequências das palavras ao longo de todas as citações dos entrevistados. As palavras "falta", "motorista", "educação", trânsito", "segurança", "ciclovia" e "desrespeito" apresentam, em ordem decrescente, expressiva participação nos argumentos dos respondentes. 
Tabela 6: Palavras mais citadas pelos entrevistados

\begin{tabular}{c|c|c}
\hline Palavras* & Quantidade** & Percentual \\
\hline Ausência & & \\
Bicicleta & 10 & $0,62 \%$ \\
Infraestrutura & 12 & $0,75 \%$ \\
Insegurança & 12 & $0,75 \%$ \\
Ciclistas & 13 & $0,81 \%$ \\
Ciclofaixas & 13 & $0,81 \%$ \\
Violência & 14 & $0,87 \%$ \\
Vias & 14 & $0,87 \%$ \\
Estrutura & 16 & $1,00 \%$ \\
Respeito & 16 & $1,00 \%$ \\
Vias & 16 & $1,00 \%$ \\
Desrespeito & 16 & $1,00 \%$ \\
Ciclovias & $\mathbf{2 1}$ & $\mathbf{1 , 3 1 \%}$ \\
Segurança & $\mathbf{2 8}$ & $\mathbf{1 , 7 4 \%}$ \\
Trânsito & $\mathbf{2 8}$ & $\mathbf{1 , 7 4 \%}$ \\
Educação & $\mathbf{3 0}$ & $\mathbf{1 , 8 7 \%}$ \\
Motoristas & $\mathbf{4 4}$ & $\mathbf{2 , 7 4 \%}$ \\
Falta & $\mathbf{5 6}$ & $\mathbf{3 , 4 8 \%}$ \\
\hline
\end{tabular}

Fonte: Dados da pesquisa (2016), com auxílio do software Atlas/TI.

*Essas palavras foram selecionadas de um total de 469 palavras citadas durante a pesquisa

**Quantidade de vezes que a palavras foi citada

No tocante às dificuldades levantadas, categorias definidas e a expressividade de algumas palavras citadas pelos respondentes, compreende-se que há apelo à mudança cultural, necessária à mudança comportamental que possibilite novos modos idealizados de existir no ambiente urbano (Heinzmann, Gubiani \& Scarpin, 2010). Com vistas a alterar padrões da condução de transporte motorizados, políticas de incentivo à mobilidade urbana com amparo em investimentos em infraestrutura e que discuta a violência, são elencadas como componentes dessa mudança cultural/comportamental. Esse apelo vai ao encontro da busca pela garantia de sobrevivência futura da população, especialmente nas grandes cidades e, consequentemente, menor impacto ambiental e melhores condições de vida/saúde. Assim, acredita-se que há maior discussão do uso racional de automóveis, com a perspectiva de adoção de políticas públicas que fomentem o uso de transporte alternativo, como a bicicleta (Silveira, 2010).

\section{CONSIDERAÇÕES FINAIS}

Diante da perspectiva de caracterizar a bicicleta como meio de transporte, desenhar o perfil dos sujeitos usuários e explorar os desafios à disseminação desse veículo como alternativa viável de transporte, identificou-se, por meio desse estudo, que há uma possível evolução na discussão sobre uso de bicicletas, impactando o público mais jovem, com a migração de um perfil masculino para um perfil mais homogêneo entre homens e mulheres, com participação de variados perfis ocupacionais, especialmente estudantes.

Atrelado ao perfil mais jovem associado ao maior uso de bicicletas, considera-se que há intensa influência da emergência das discussões sobre sustentabilidade, em especial, no âmbito educacional, amparada pela abordagem da educação ambiental. Ao mesmo tempo, aos mais jovens se atribui uma construção identitária incipiente, que possibilitaria maior flexibilidade às mudanças de hábitos e novas elaborações sobre a relação sujeito-ambiente que podem, também, favorecer a adoção de comportamentos pró-ambientais. Nesse sentido, a análise por cluster colabora com a elaboração de políticas públicas para a Mobilidade Urbana Sustentável que considere a segmentação dos usuários, de forma que se pense em ações mais aderentes a cada perfil, ou sensibilização, com amparo nos valores de cada cluster. 
Este contingente pode ser considerado um indício de que o cuidado com o meio ambiente e com a saúde da cidade pode ser estimulado, pois há uma tendência entre jovens pela diminuição do veículo motorizado, implicando em uma diminuição das emissões de gases tóxicos, como o $\mathrm{CO}_{2}$, e da poluição sonora, podendo favorecer mobilidade urbana mais sustentável. Pedalar favorece a boa convivência e influencia a prática saudável da população, fazendo do espaço urbano uma plataforma para a relação sustentável entre as pessoas e a cidade.

No que diz respeito aos principais empecilhos à adesão ao modo cicloviário, a mudança cultural emerge como ponto de maior problematização, o que confirma estudos que abordam a insegurança e a falta de educação (Maia \& Moreira, 2010; Pezutto \& Sanches, 2004), e a necessária prioridade nas políticas de mobilidade urbana para a estruturação viária adequada ao uso de bicicletas (Silveira \& Maia, 2015), à medida que é identificado, também, como um meio de locomoção que agrega saúde à vida das pessoas (Providelo; Sanches, 2010).

Os resultados da pesquisa permitem inferir que a cultura de mobilidade urbana na Cidade de Fortaleza se encontra em expansão, entretanto, enseja um planejamento de políticas públicas visando à mobilidade urbana de forma sustentável, objetivando a melhoria do comportamento dos motoristas frente aos usuários de bicicleta.

Acredita-se no potencial de pesquisas que explorem a Mobilidade Urbana Sustentável sob o prisma dos valores pessoais atribuídos ao uso de bicicleta, o que possibilitaria uma ampliação da pesquisa, à medida que aborde aspectos culturais e de apropriação do espaço na delimitação de perfis. Ainda, sugere-se ampliação do estudo em outros centros urbanos numa perspectiva de ampliação dos achados e, também, comparação entre cidades.

\section{REFERÊNCIAS}

Albuquerque, C. de, Vicentini, J. de O., \& Pipitone, M. A. P. (2015) O júri simulado como prática para a educação ambiental crítica. Rev. Bras. Estud. Pedagog., Brasília, 96(242),199-215, abril.

Amorim Filho, O., \& Serra, R. V. (2001) Evolução e perspectivas do papel das cidades médias no planejamento urbano e regional. In Cidades médias brasileiras. Rio de Janeiro: Ipea.

Ary, M. B.(2009) Avaliação da qualidade das ciclovias de Fortaleza. Anais do Congresso Brasileiro de Transporte e Trânsito, Curitiba, PR, Brasil, 17.

Azevedo, C. E. F., Oliveira, L. G. L., Gonzalez, R. K., \& Abdalla, M. M. (2013) A estratégia de triangulação: objetivos, possibilidades, limitações e proximidades com o pragmatismo. Anais do Encontro de Ensino e Pesquisa em Administração e Contabilidade, Brasília, BR, Brasil, 4.

Azevedo Filho, M. A. N. (2012) Análise do processo de planejamento dos transportes como contribuição para a mobilidade urbana sustentável. Tese doutorado, Universidade de São Paulo, São Carlos, SP, Brasil.

Banerjee, S. B. (2008) Corporate social responsibility: the good, the bad and the ugly. Critical Sociology, 34(1), 51-79.

Barbieri, J. C. (1997) Políticas públicas indutoras de inovações tecnológicas ambientalmente saudáveis nas empresas. Revista Brasileira de Administração Pública, 31(2), 135-152.

Bardin, L. (2011) Análise de conteúdo. São Paulo: Edições 70.

Bertini, R. L. (2005) You are the traffic jam: an examination of congestion measures. Anais do Meeting of the Transportation Research Board, Washington, DC, Estados Unidos, 85. 
Bezerra, B. S., Kaiser, I. M., \& Battistelle, R. A. G. (2015) Road safety implications for sustainable development. Latin American Journal of Management for Sustainable Development, 2, 1-17.

Bomfim, Z. A. C. (2003) Cidade e afetividade: estima e construção dos mapas afetivos de Barcelona e São Paulo. Tese doutorado, Pontifícia Universidade Católica de São Paulo, São Paulo, SP, Brasil.

Castro, C. M. S. E., Barbosa, H. M., \& Oliveira, L. K. de. (2013) Análise do potencial de integração da bicicleta com o transporte coletivo em Belo Horizonte. J. Transp. Lit., Manaus, 7(2), 146-170, abril.

Costa, F. J. da, Ramos, R. R., Ramos, I. M. M., \& Oliveira, L. G. L. (2013) Valores pessoais e gestão socioambiental: um estudo com estudantes de administração. RAM, Revista de Administração Mackenzie, São Paulo, 14(3), 183-208, maio/junho.

Curtis, C., \& Low, N. (2012) Institutional barriers to sustainable transport. Ashgate, Farnham, Surrey; Burlington, VT.

Downs, A. (2004) Still stuck in traffic: coping with peak-hour congestion. Washington: BrookingsIntitution Press.

Franco, L. P. C. (2012) Perfil e demanda dos usuários de bicicletas em viagens pendulares. Tese de doutorado, Instituto Militar de Engenharia, Rio de Janeiro, RJ, Brasil.

Gomide A. A., \& Morato, R. (2011) Instrumentos de desestímulo ao uso do transporte individual motorizado: lições e recomendações. São Paulo: Instituto de Energia e Meio Ambiente.

Gomide, A. A. (2008) Agenda governamental e o processo de políticas públicas: o projeto de lei de diretrizes da política nacional de mobilidade urbana. Texto para Discussão - Ipea, Brasília: Ipea, 1334.

Heinzmann, L. M., Gubiani, C. A., \& Scarpin, J. E. (2010) Cultura organizacional: classificação de artigos do EnAnpadEnGPR sob o enfoque de Smircich (1983) e Freitas (1991b). Revista da Universidade Estadual do Oeste do Paraná, 9(16).

Hidalgo, D. (2011) Transporte Sostenible para América Latina: situaciónactual y perspectivas. Documento de respaldo foro de transportes sostenible FTS de América Latina 2011, Bogotá: Centro de las Naciones Unidas para El Desarrollo Regional Uncrd, Banco Interamericano de Desarrollo BID, junio.

Hillel, O. (2008) Metrópoles não são vilãs ambientais. Entrevista para revista Mega Cidades, Oesp, agosto.

Hopkins, C. (2012) Twenty years of education for sustainable development. Journal of Education for Sustainable Development, 6(1).

Ipea. Instituto de Pesquisa Econômica Aplicada. (2011) Sistema de indicadores de percepção social mobilidade urbana. Brasília: Ipea.

Lynch, K. (1998) La imagen de la cidad. Barcelona: Editorial Gustavo Gil. 
Magalhães, J. R. L., Campos, V. B. G., \& Bandeira, R. A. de M. (2015) Metodologia para identificação de redes de rotas cicláveis em áreas urbanas. J. Transp. Lit., Manaus, 9(3), 35-39, setembro.

Maia, C. M., \& Moreira, M. E. P. (2010) Caracterização dos deslocamentos de ciclistas e fatores que influenciam suas viagens em Fortaleza - CE. Anais do Congresso de Pesquisa e Ensino em Transportes, Salvador, BA, Brasil, 26.

Maxwell, J. A. (1996) Qualitative research design: an interactive approach. Thousand Oaks: Sage.

Mebratu, D. (1998) Sustainability and sustainable development: historical and conceptual review. Environ Impact Asses Rev., 18, 493-520.

Ministério das Cidades (2007) Plano de Mobilidade por Bicicleta nas Cidades. Brasília: Secretaria Nacional de Transporte e da Mobilidade Urbana, 2007. (Coleção Bicicleta Brasil, Caderno de referência para elaboração de Plano de Mobilidade por Bicicleta nas Cidades).

Montiel, I. (2008) Corporate social responsibility and corporate sustainability: separate past, common futures. Organization \& Environment, 21(3), 245-269.

Moser, G. (2005) A psicologia ambiental: competência e contornos de uma disciplina. Psicologia USP, 16(1), 279-294.

Oliveira, C. M. de, Misoczky, M. C. (2016). Urban entrepreneurialism in FIFA Word Cup host cities: the case of Porto Alegre. Organizações \& Sociedade, 23 (79), 624-645.

Pezzuto, C. C., \& Sanches, S. P. (2004) Identificação dos fatores que influenciam no uso da bicicleta. Anais do Congresso de Pesquisa e Ensino em Transportes, Florianópolis, SC, Brasil, 18.

Plume.(2013) Synthesis report on urban sustainability and its appraisal. Planning for Urban Mobility in Europe. Local: Plume.

PMF. Prefeitura Municipal de Fortaleza. (2015) Cartilha: cidadania sustentável. Fortaleza: Secretaria Municipal de Cidadania e Direitos Humanos.

Pohlmann, M. C. (2009) Análise de conglomerados. In L. J. Corrar, E. Paulo, \& J. M. Dias Filho. Análise multivariada: para os cursos de Administração, Ciências Contábeis e Economia. São Paulo: Atlas.

Providelo, J. K., \& Sanches, S. P. (2010) Percepções de indivíduos acerca do uso da bicicleta como modo de transporte. Transportes, 2, 53-61, junho.

Resende, P. T. V., \& Sousa, P. R. (2009) Mobilidade urbana nas grandes cidades brasileiras: um estudo sobre os impactos do congestionamento. Anais do Simpósio de Administração da Produção, Logística e Operações Internacionais, São Paulo, SP, Brasil.

Ross, S.L., \& Yinger, J. (2000) Timing equilibria in an urban model with congestion. Journal of Urban Economics, 47(3), May. 
Saldanha, L., Fraga, C., \& Santos, M. P. de S. (2015) Discussões preliminares sobre serendipidade, bicicleta e turismo envolvendo a Ilha de Paquetá no Rio de Janeiro (RJ). Caderno Virtual de Turismo, 15(3), 379-389, dezembro.

Seabra, L. O. P. W. G., Taco, W. G. M., \& Dominguez, E. M. (2013) Sustentabilidade em transportes: do conceito às políticas públicas de mobilidade urbana. Revista dos Transportes Públicos - Antp, 35(134), 103-124.

Silveira, M. O. (2010) Mobilidade sustentável: a bicicleta como meio de transporte integrado. Dissertação de mestrado, Universidade Federal do Rio de Janeiro, Rio de Janeiro, RJ, Brasil.

Silveira, M. O., \& Maia, M. L. A. (2015) Variáveis que influenciam no uso da bicicleta e as crenças da Teoria do Comportamento Planejado. Revista Transportes, 23(1).

Sinay, M. C. F. de, Dalbem, M. C., Loureiro, I. A., \& Vieira, J. D. M. (2013) Ensino e pesquisa em gestão ambiental nos programas brasileiros de pós-graduação em administração. RAM, Revista de Administração Mackenzie, São Paulo, 14(3), 55-82, junho.

Soares, A. G., Guth, D., Amaral, J. P., \& Maciel, M. (Org.). (2015) A bicicleta no Brasil 2015. São Paulo: D. Guth.

Sulaiman, S. N. (2011). Educação ambiental, sustentabilidade e ciência: o papel da mídia na difusão de conhecimentos científicos. Ciência e Educação, Bauru, 17(3).

TCU. Tribunal de Contas da União. (2010) Relatório e parecer prévio sobre as contas do Governo da República. Brasília: Tribunal de Contas da União. (Exercício 2010.).

Un-Habitat. (2013) Planning and design for sustainable urban mobility: policy directions. United Nations Human Settlements Programme, Nairobi: Earths can from Routledge, Abingdon.

Valdanha Neto, D., Kawasaki, C. S. (2015) A temática ambiental em documentos curriculares nacionais do ensino médio. Ens. Pesqui. Educ. Ciênc., Belo Horizonte, 17(2), 483-499, agosto.

Vieira, H., Valente, A. M., Pegas, H., Moreira, M. E., \& Oliveira, A. M. (2010) O planejamento cicloviário: a busca da sustentabilidade a partir de erros e sucesso dos outros. Anais do Congresso Luso-brasileiro para o Planejamento Urbano Regional Integrado Sustentável, Portugal, 4.

Zulkifli, I., Norma, M. T. C., Chong, C. H., \& Loh, T. C. (2001) The effects of crating and road transportation on stress and fear responses of broiler chickens treated with ascorbic acid. Archivfur Gefluge lkunde, 65, 33-37.

Data da submissão: 19/10/2016

Data de aceite: 17/03/2017 